Pacific Journal of Mathematic 


\title{
ON ALGEBRAS WHOSE FACTOR ALGEBRAS ARE BOOLEAN
}

\author{
J. M. G. FELl AND Alfred TARSKI
}

1. Introduction. ${ }^{1}$ We consider an algebraic system $\mathscr{A}=\langle A,+\rangle$ constituted by an arbitrary set $A$ and a binary operation + . The set $A$ is assumed to be closed under + , and to contain a (uniquely determined) zero element, that is, an element 0 such that

$$
x+0=0+x=x
$$

for every $x$ in $A$. We shall refer to such a system simply as an algebra. By a subalgebra $B$ of 20 we understand an arbitrary subset of $A$ which is closed under + and contains 0 as an element.

For two subalgebras $B$ and $C$, a function $f$, whose domain includes (but does not necessarily coincide with) $B$ and which maps $B$ onto a subset of $C$ in such a way that

$$
f\left(b_{1}+b_{2}\right)=f\left(b_{1}\right)+f\left(b_{2}\right)
$$

for all $b_{1}, b_{2}$ in $B$, is called as usual a $(B, C)$-homomorphism; if in addition $f$ is biunique on $B$, and maps $B$ onto the whole of $C$, it is called a $(B, C)-i$ somorphism.

A relation $R$ holding between certain pairs of elements of $A$ is called a congruence relation over $2 Y$ if (i) $R$ is an equivalence relation whose field is $A$, and (ii) $(a+b) R\left(a^{\prime}+b^{\prime}\right)$ whenever $a R a^{\prime}$ and $b R b^{\prime}$; here we have expressed symbolically by $c R d$ the statement that $R$ holds between the elements $c$ and $d$. Suppose that $R$ is a congruence relation over $\mathscr{U}$. For each element $a$ of $A$, the coset of $a$ under $R$, in symbols $a / R$, will be defined as the set of all $b$ in $A$ for which $a R b$. It is easy to see that any two cosets are either identical or else have no element in common, and that the set-theoretical union of all the cosets is simply $A$. If $a / R$ and $b / R$ are any two cosets, we denote by $a / R+{ }^{\prime} b / R$ the coset $(a+b) / R$. By condition (ii) of the definition of a congruence relation, this determines an operation $+^{\prime}$ on pairs of cosets. The algebra consisting of

\footnotetext{
${ }^{1} \mathrm{~A}$ detailed discussion of all the notions and results contained in the Introduction will be found in [8] (see in particular Appendix $\oint \oint A$ and $B$ ) and [4] (see in particular $\$ \$ 1$ and 2 ).
}

Received July 3, 1951.

Pacific J. Math. 2 (1952), $297-318$ 
the family of all cosets of elements of $A$ under $R$, with the binary operation +', is called the coset algebra (of $\mathscr{A}$ under $R$ ) and is denoted by $\mathscr{X} / R .^{2}$

Given a subalgebra $B$ of 2 , by the center of $B$-in symbols $B^{c}$-we mean the set of all $c$ in $B$ satisfying the following conditions: (i) there is an element $d$ in $B$ with $c+d=0$; (ii) the commutative-associative formula

$$
\left(b_{1}+b_{2}\right)+c=b_{1}+\left(b_{2}+c\right)=\left(b_{1}+c\right)+b_{2}
$$

holds for any $b_{1}, b_{2}$ in $B .{ }^{3}$ Clearly 0 belongs to the center of all subalgebras. It is not hard to see that $b+c=c+b$ whenever $b \in B$ and $c \in B^{c}$ (commutative law); and that $b_{1}+c=b_{2}+c$ implies $b_{1}=b_{2}$ for all $b_{1}, b_{2}$ in $B$ and $c$ in $B^{c}$ (cancellation law). It follows that for any subalgebra $B$, the system $\left(B^{c},+\right)$ is an Abelian group. A subalgebra $B$ in which $B^{c}=\{0\}$, that is, $B^{c}$ consists only of 0 , is called centerless. Similarly, an algebra $\mathscr{A}=\langle A,+\rangle$ in which $A^{c}=\{0\}$ is called centerless.

A subalgebra $D$ is called the direct produet of subalgebras $B$ and $C$ if (i) every element $d$ in $D$ can be uniquely represented in the form $d=b+c$, where $b \in B$ and $c \in C$; (ii) we have $b+c \in D$ for every $b$ in $B$ and $c$ in $C$; (iii) the formula

$$
\left(b_{1}+c_{1}\right)+\left(b_{2}+c_{2}\right)=\left(b_{1}+b_{2}\right)+\left(c_{1}+c_{2}\right)
$$

holds for arbitrary $b_{1}, b_{2}$ in $B$, and $c_{1}, c_{2}$ in $C$. The subalgebra $D$ with these properties (if it exists at all) is of course uniquely determined by $B$ and $C$, and will be denoted by $B \times C$. It is clear that if $B \times C$ exists, and $B_{1}$ and $C_{1}$ are subalgebras included respectively in $B$ and $C$, then $B_{1} \times C_{1}$ exists. It can be shown without difficulty that the operation $\times$ on pairs of subalgebras satisfies the commutative and associative laws. Two subalgebras $B$ and $C$ of 21 are called complementary factors if $B \times C=A$, and a subalgebra $B$ which has at least one complementary factor is referred to as a factor of 2 . For complementary factors $B$ and $C$ of $\mathscr{A}$, it follows from the definition of direct product that $b+c=c+b$ whenever $b \in B$ and $c \in C$; and $a_{1}+\left(a_{2}+a_{3}\right)=\left(a_{1}+a_{2}\right)+a_{3}$ whenever two of the elements $a_{1}, a_{2}, a_{3}$ belong to one of the pair $B$ and $C$, while the remaining element belongs to the other; it also follows that there exist an $(A, B)$-homomorphism $f$ and an $(A, C)$-homomorphism $g$ such that $a=f(a)$ $+g(a)$ for every $a$ in $A$.

${ }^{2}$ This definition of a coset algebra is given in [8], p. 79 and p. 175. For an introduction to equivalence relations and congruence relations, see [3], pp. 159-162.

${ }^{3}$ This definition of center is given in [5]. It can easily be shown to be equivalent to the definition given in [4], p. 24, when the latter is applied to algebras with one binary operation. 
The notion of direct product can be extended in an obvious way to any finite number of subalgebras.

In order to discuss direct decompositions of the algebra $\mathscr{A}$, that is, the representations of $A$ as a direct product of subalgebras, it is important to study algebraic properties of the family $\mathfrak{F}(\mathfrak{2})$ of all factors of 2 . In particular, it proves convenient to consider the so-called factor algebra of $\mathscr{2}$, that is, the algebraic system formed by the family $\mathfrak{F}(\mathscr{U})$ and the operation $x_{\text {. }}$ (This is clearly a system in which the closure property does not hold.) An especially simple case is the one in which the system $\langle\mathcal{F}(\mathscr{Y}), x\rangle$ is what is called a disjunctive Boolean algebra, that is, in which the operation $\times$ has all the properties of Boolean-algebraic addition (join operation) restricted to couples of disjoint elements. ${ }^{4}$ Instead of the term 'disjunctive Boolean algebra', we shall use the simpler term 'Boolean algebra'. Among consequences which follow from the assumption that the factor algebra is Boolean, we mention the following: (i) the factor algebra of $2 \mathbb{C}$ has the so-called refinement property; (ii) $A$ has, apart from order, at most one representation as a direct product of so-called indecomposable subalgebras; and (iii) it has just one such representation if it is finite. Various conditions are known which are necessary and sufficient for the factor algebra $\langle\mathcal{F}(\mathscr{2}), x\rangle$ to be Boolean. ${ }^{5}$ One such condition is that $\mathfrak{F}(\mathscr{Y})$ be a Boolean algebra in the usual sense under set-theoretical inclusion. Another condition, which will actually be used below, is the distributive law for the factor algebra in the following form:

If $B, C_{1}$ and $C_{2}$ are factors of $\mathscr{A}$, and if $C_{1} \times C_{2}$ exists and is also a factor of 2 , then

$$
B \cap\left(C_{1} \times C_{2}\right)=\left(B \cap C_{1}\right) \times\left(B \cap C_{2}\right),
$$

(where $B \cap C$ denotes as usual the intersection of the sets $B$ and $C$ ).

The main purpose of this paper is to establish two further necessary and sufficient conditions of the same kind; they will be given in $\$ 2$, Theorem 3 and Corollary 4. In particular, from Theorem 3 we shall see that a necessary and sufficient condition for the factor algebra to be Boolean is that for every factor $B$ there exists exactly one factor complementary to $B$. In $\S 3$ we shall see how, by application of the results of $\$ 2$, various simple and interesting classes of algebraic systems can be shown to have Boolean factor algebras. Finally, in $\$ 4$, an extension of the results obtained to algebraic systems with many operations will be briefly discussed.

\footnotetext{
${ }^{4}$ See [8], p. $205 \mathrm{ff}$.

${ }^{5}$ See $[8]$, p. $272 \mathrm{ff}$.
} 
2. Main Results. We start with two auxiliary theorems.

Theовем 1. Let $A=B \times C$, and let $D$ be a subalgebra of 2 . In order that

$$
A=B \times D,
$$

it is necessary and sufficient that there be a $\left(C, B^{c}\right)$-homomorphism $f$ such that $D$ consists of all elements of the form $c+f(c)$ with $c$ in $C$.

Proof. Part I (proof of necessity). ${ }^{6}$ Assume

$$
A=B \times D .
$$

Since by hypothesis

$$
A=B \times C,
$$

there is (see Introduction) an $(A, B)$-homomorphism $g$ and an $(A, C)$-homomorphism $h$ such that

$$
a=g(a)+h(a) \quad \text { for every } a \text { in } A .
$$

Obviously $h$ is also a $(D, C)$-homomorphism. With the aid of (1) and (2) we can even show that

$$
h \text { is a }(D, C) \text { - isomorphism. }{ }^{7}
$$

Therefore, denoting by $k$ the inverse of the biunique function obtained by restricting the domain of $h$ to $D$, we can put, for each $c$ in $C$,

$$
f(c)=g[k(c)] \text {. }
$$

Evidently

$$
f \text { is a }(C, B) \text { - homomorphism. }
$$

By (3) and (5),

$$
k(c)=c+f(c) \text { for all } c \text { in } C .
$$

From this and (4) we deduce that

${ }^{6}$ The proof of the "necessity part" of our Theorem 1 is essentially contained in the proof of Theorem 2.17, p. 32 of [4]. Also if, instead of considering arbitrary algebras, we restrict ourselves to the so-called loops, then the "necessity part" of our Theorem 1 can easily be derived from Corollary 2, p. 69 of [1]; on the other hand, by analyzing the proof of Theorem 1, we can see that the corollary just mentioned extends from loops to arbitrary algebras.

${ }^{7}$ See [4], p. 32, the proof of Theorem 2.17. 
$D$ consists of all elements of the form $c+f(c)$ with $c$ in $C$.

Let us fix an element $c$ of $C$. By (6) and (7),

$$
f(c) \in B \text { and } c+f(c) \in D \text {. }
$$

By (1) there are elements $b$ of $B$ and $d$ of $D$ such that $c=d+b$. But $d$ has the form $c_{1}+f\left(c_{1}\right)$, with $c_{1}$ in $C$. Consequently, by the associative law for elements of complementary factors (stated in the Introduction),

$$
c=\left[c_{1}+f\left(c_{1}\right)\right]+b=c_{1}+\left[f\left(c_{1}\right)+b\right]
$$

from which it follows by the condition of unique representation in the definition of direct product that $c=c_{1}$ and $f\left(c_{1}\right)+b=0$, and hence

$$
f(c)+b=0 \text {. }
$$

Let $b_{1}, b_{2} \in B$. By applying again the associative as well as the commutative law for complementary factors, we obtain from (1), (2), and (8):

$$
\begin{aligned}
\left\{b_{1}\right. & \left.+\left[b_{2}+f(c)\right]\right\}+c=b_{1}+\left\{\left[b_{2}+f(c)\right]+c\right\} \\
& =b_{1}+\left\{b_{2}+[c+f(c)]\right\}=\left\{b_{1}+[c+f(c)]\right\}+b_{2} \\
& =\left\{\left[b_{1}+f(c)\right]+c\right\}+b_{2}=\left[b_{1}+f(c)\right]+\left(c+b_{2}\right) \\
& =\left\{\left[b_{1}+f(c)\right]+b_{2}\right\}+c,
\end{aligned}
$$

so that finally

$$
\left\{b_{1}+\left[b_{2}+f(c)\right]\right\}+c=\left\{\left[b_{1}+f(c)\right]+b_{2}\right\}+c .
$$

Hence, by observing that $b_{1}+\left[b_{2}+f(c)\right]$ and $\left[b_{1}+f(c)\right]+b_{2}$ are in $B$, while $c \in C$, we conclude from (2) that

$$
b_{1}+\left[b_{2}+f(c)\right]=\left[b_{1}+f(c)\right]+b_{2} .
$$

Similarly,

$$
b_{1}+\left[b_{2}+f(c)\right]=\left(b_{1}+b_{2}\right)+f(c) \text {. }
$$

According to the definition of $B^{c}$, (9), (10), and (11) assure us that $f(c) \in B^{c}$ whenever $c \in C$. Therefore, by (6),

$$
f \text { is a }\left(C, B^{c}\right) \text {-homomorphism. }
$$

By (7) and (12) the proof of necessity is complete. 
Part II (proof of sufficiency). Assume that a function $f$ and a set $D$ are given which satisfy (7) and (12).

Let $a \in A$. We may write $a=c+b$, where $b \in B$ and $c \in C$. Since $f(c)$ $\in B^{c}$, there is a $b_{1}$ in $B$ such that $f(c)+b_{1}=0$. Therefore, applying the associative properties of elements in the center and in complementary factors, we get:

$$
a=c+\left\{\left[f(c)+b_{1}\right]+b\right\}=c+\left[f(c)+\left(b_{1}+b\right)\right]=[c+f(c)]+\left(b_{1}+b\right),
$$

where of course $c+f(c) \in D$ and $b_{1}+b \in B$. Thus every element $a$ of $A$ is of the form

$$
a=d^{\bullet}+b^{\prime} \text {, where } d^{\prime} \in D \text { and } b^{\prime} \in B .
$$

Suppose that $d+b=d^{\prime}+b^{\prime}$, where $d, d^{\prime} \in D$, and $b, b^{\prime} \in B$. By hypothesis, $d$ and $d^{\prime}$ are of the forms respectively $c+f(c)$ and $c^{\prime}+f\left(c^{\prime}\right)$, where $c, c^{\prime} \in C$. Therefore

$$
c+[f(c)+b]=[c+f(c)]+b=\left[c^{\prime}+f\left(c^{\prime}\right)\right]+b^{\prime}=c^{\prime}+\left[f\left(c^{\prime}\right)+b^{\prime}\right] .
$$

From this follows $c=c^{\prime}$, hence $d=d^{\prime}$; also

$$
f(c)+b=f(c)+b^{\prime} .
$$

Hence, $f(c)$ being in $B^{c}$, we obtain, using the commutative law and cancellation law for elements in the center, $b=b^{\prime}$. Therefore:

$$
\text { The representation (13) of an arbitrary } a \text { in } A \text { is unique. }
$$

It is furthermore obvious that

$$
d+b \in A \text { for every } d \text { in } D \text { and } b \text { in } B .
$$

Finally, suppose $d, d^{\circ} \in D$, and $b, b^{\circ} \in B$. As usual, $d=c+f(c)$ and $d^{\prime}=c^{\prime}+f\left(c^{\prime}\right)$, where $c, c^{\prime} \in C$. Then, applying various properties of the center and direct product which were used earlier in this proof, we obtain

$$
\begin{aligned}
\left(d+d^{\prime}\right)+\left(b+b^{\prime}\right) & =\left\{[c+f(c)]+\left[c^{\prime}+f\left(c^{\prime}\right)\right]\right\}+\left(b+b^{\prime}\right) \\
& =\left\{\left(c+c^{\prime}\right)+\left[f(c)+f\left(c^{\prime}\right)\right]\right\}+\left(b+b^{\prime}\right) \\
& =\left(c+c^{\prime}\right)+\left\{\left[f(c)+f\left(c^{\prime}\right)\right]+\left(b+b^{\prime}\right)\right\} \\
& =\left(c+c^{\prime}\right)+\left\{[f(c)+b]+\left[f\left(c^{\prime}\right)+b^{\prime}\right]\right\} \\
& =\{c+[f(c)+b]\}+\left\{c^{\prime}+\left[f\left(c^{\prime}\right)+b^{\prime}\right]\right\} \\
& =\{[c+f(c)]+b\}+\left\{\left[c^{\prime}+f\left(c^{\prime}\right)\right]+b^{\prime}\right\},
\end{aligned}
$$


so that finally

$$
\left(d+d^{\prime}\right)+\left(b+b^{\prime}\right)=(d+b)+\left(d^{\prime}+b^{\prime}\right) \text { for } b, b^{\prime} \text { in } B \text { and } d, d^{\prime} \text { in } D \text {. }
$$

From (13), (14), (15), and (16) we conclude that

$$
A=B \times D
$$

and the proof of sufficiency is complete.

Corollary 2. Let $A=B \times C$. For $C$ to be the only complementary factor of $B$, it is necessary and sufficient that the only $\left(C, B^{c}\right)$-homomorphism (with domain restricted to $C$ ) be the constant function $f$, with $f(c)=0$ for every $c$ in $C$.

Proof. The sufficiency follows immediately from Theorem 1.

To prove necessity, ass ume that $C$ is the only complement of $B$, and that $g$ is a $\left(C, B^{c}\right)$-homomorphism. For any given $c$ in $C$, by Theorem 1 , we have $c+g(c)$ $\in C$, and of course also $[c+g(c)]+0=c+g(c)$. Hence, by the hypothesis of the theorem and the definition of direct product, we have $g(c)=0$.

THEOREM 3. For the factor algebra of an algebra 24 to be Boolean, it is necessary and sufficient that every factor of \&2 have exactly one complementary factor. ${ }^{8}$

Proof. The necessity follows from well-known properties of Boolean algebras.

To prove sufficiency, assume that every factor of $\mathscr{U}$ has a unique complementary factor. Consider three subalgebras $B, C_{1}, C_{2}$ of थr such that

$$
B, C_{1}, C_{2} \text { and } C_{1} \times C_{2} \text { are factors of } \mathscr{Q} \text {. }
$$

Then, for some subalgebras $D$ and $C_{3}$, we have

$$
A=B \times D=\left(C_{1} \times C_{2}\right) \times C_{3},
$$

and it is known that formula (2) implies

$$
B \subseteq\left\{\left[\left(D^{c} \times B\right) \cap C_{1}\right] \times\left[\left(D^{c} \times B\right) \cap C_{2}\right]\right\} \times\left[\left(D^{c} \times B\right) \cap C_{3}\right] .^{9}
$$

In view of (2), there is an $\left(A, C_{1}\right)$-homomorphism $g_{1}$, an $\left(A, C_{2}\right)$-homomorphism $g_{2}$, and an $\left(A, C_{3}\right)$-homomorphism $g_{3}$ such that

${ }^{8}$ This theorem seems to be related to a result in [2], according to which a lattice possessing (relative) complements is a Boolean algebra if and only if all complements are unique. We see, however, no way of applying the result just mentioned in the proof of Theorem 3.

${ }^{9}$ See [4], p. 21, Theorem 2.9. 


$$
a=\left[g_{1}(a)+g_{2}(a)\right]+g_{3}(a) \text { for all } a \text { in } A
$$

(see Introduction). Hence it follows by (3) that

$$
g_{1}(b) \in D^{c} \times B, g_{2}(b) \in D^{c} \times B \text {, and } g_{3}(b) \in D^{c} \times B \text { for all } b \text { in } B .
$$

Similarly by (2) there is an $(A, B)$-homomorphism $f_{1}$ and an $(A, D)$-homomorphism $f_{2}$ such that

$$
a=f_{1}(a)+f_{2}(a) \text { for all } a \text { in } A .
$$

Clearly $f_{2} g_{1}$ is an $(A, D)$-and hence also a $(B, D)$-homomorphism. Moreover, if $b \in B$, we have, by (6),

$$
g_{1}(b)=f_{1} g_{1}(b)+f_{2} g_{1}(b) \text {, where } f_{1} g_{1}(b) \in B \text { and } f_{2} g_{1}(b) \in D .
$$

By comparing (5) and (7), we easily see that $f_{2} g_{1}(b) \in D^{c}$, so that $f_{2} g_{1}$ proves to be a $\left(B, D^{c}\right)$-homomorphism. But, by hypothesis, $B$ is the unique factor complementary to $D$. Therefore, by Corollary $2, f_{2} g_{1}(b)=0$; hence, by (7),

$$
g_{1}(b) \in B \text { for all } b \text { in } B \text {. }
$$

For entirely analogous reasons,

$$
g_{2}(b) \in B \text { for all } b \text { in } B .
$$

Evidently $\left(B \cap C_{1}\right) \times\left(B \cap C_{2}\right)$ exists. By use of (4), (8), and (9) we obtain

$$
B \cap\left(C_{1} \times C_{2}\right) \subseteq\left(B \cap C_{1}\right) \times\left(B \cap C_{2}\right) .
$$

But it is clear that $\left(B \cap C_{1}\right) \times\left(B \cap C_{2}\right) \subseteq B \cap\left(C_{1} \times C_{2}\right)$. Consequently

$$
B \cap\left(C_{1} \times C_{2}\right)=\left(B \cap C_{1}\right) \times\left(B \cap C_{2}\right) .
$$

We have now shown that, whenever $B, C_{1}, C_{2}$, and $C_{1} \times C_{2}$ are factors of $\mathfrak{u}$, the relation $(10)$ holds. As was pointed out in the Introduction, this is a sufficient condition for the factor algebra of $\mathfrak{U}$ to be Boolean.

CoROllary 4. For the factor algebra of an algebra at to be Boolean, it is necessary and sufficient that, for every pair $B$ and $C$ of complementary factors of $\mathfrak{A}$, the only $\left(C, B^{c}\right)$-homomorphism (with domain restricted to $C$ ) be the constant function $f$ such that $f(c)=0$ whenever $c \in C$.

Proof. This follows from Corollary 2 and Theorem 3.

We should like to conclude this section with some remarks which are related 
to Theorem 3, even though they are not connected with the main topic of this paper. By Theorem 3, in every algebra \&2t whose factor algebra is not Boolean, we can find two different factors $B$ and $C$ which have a common complement $D$. Hence it appears natural to consider the relation which holds between any two factors $B$ and $C$ if and only if $B \times D=C \times D=A$ for some factor $D$. This relation is often involved in the discussion of direct decompositions of algebras and some of its properties can be found in the literature; it is known, for instance, that any two factors between which this relation holds are isomorphic (and even what is called central-isomorphic). ${ }^{10}$ In this connection it may be interesting to notice that the relation in question, as opposed to that of isomorphism, is not transitive, and hence not an equivalence relation. To demonstrate this fact, we take for a counterexample the additive group $\mathscr{A}=\langle A,+\rangle$ of complex numbers $a+b i$, where $a$ and $b$ are integers. If $p$ is an element of $A$, we shall denote by $[p]$ the subgroup of 21 generated by the element $p$. Now let $B_{1}=$ $[i], B_{2}=[3+2 i], C_{1}=[1], C_{2}=[1+i], C_{3}=[7+5 i]$. It is easy to check that

$$
A=B_{1} \times C_{1}=B_{1} \times C_{2}=B_{2} \times C_{2}=B_{2} \times C_{3} .
$$

Let $\approx$ be the relation which holds between factors $B$ and $C$ if and only if $B \times D=$ $C \times D=A$ for some $D$. Then, by (1),

$$
C_{1} \approx C_{2} \text { and } C_{2} \approx C_{3} \text {. }
$$

Now, suppose $C_{1} \approx C_{3}$. Then there is a factor $D$ of 21 such that $A=D \times C_{1}=D \times$ $C_{3}$. Using (1), we observe that $D \approx B_{1}$; therefore $D \cong B_{1}$, and $D$ is an infinite cyclic group. Let $a, b$ be such a pair of integers that $D=[a+b i]$. In order that $D \times C_{1}=A$, it is necessary that

$$
\left|\begin{array}{ll}
a & 1 \\
b & 0
\end{array}\right|= \pm 1,
$$

from which follows $b= \pm 1$. Similarly, in order that $D \times C_{3}=A$, it is necessary that

$$
\left|\begin{array}{cc}
a & 7 \\
b & 5
\end{array}\right|= \pm 1 \text {; }
$$

\footnotetext{
${ }^{10}$ See [4], p. 32, Theorem 2.17, and [1], p. 69, Corollary 2.
} 
this, combined with $b= \pm 1$, gives $5 a \pm 7= \pm 1$, an impossibility. Consequently it is not true that $C_{1} \approx C_{3}$.

Now (2) and (3) imply at once that $\approx$ is not transitive.

3. Applications. We shall now apply the results of the preceding section to various special classes of algebraic systems. Although many of the results we shall formulate in the present section are not essentially new, it may be interesting to see that all of them can now be established by means of a simple and uniform method.

While most of our discussion will be based directly on Corollary 4, we shall start with a simple case of a straight application of Theorem 3.

THEOREM 5. The factor algebra of an arbitrary cyclic group is Boolean. ${ }^{11}$

Proof. Let \& be a cyclic group of finite order $n$. As is well known, \& has at most one subgroup of any given order. If now $B$ is a factor of $B$ of order $m$, then a complementary factor of $B$ must have exactly $n / m$ elements, and hence is uniquely determined. Hence, by Theorem 3, the conclusion follows immediately. The theorem applies trivially to infinite cyclic groups, since they are known to be indecomposable.

Theorem 5 can be considerably strengthened if we introduce the notion of a generalized cyclic group. A generalized cyclic group is a group \& any two elements of which are members of the cyclic group generated by some third element of $\$$. All generalized cyclic groups are Abelian, and finite generalized cyclic groups are cyclic. Now it is a known result that the lattice of all subgroups of a group $\&$ is distributive if and only if $\&$ is a generalized cyclic group. From this it follows that the factor algebra of an arbitrary generalized cyclic group is Boolean. The latter fact also follows easily from our Corollary 4.

Since the above-mentioned result on lattices of subgroups holds in both directions, one might enquire whether or not the statement on factor algebras of generalized cyclic groups admits a converse. One can indeed show easily from the well-known structure of finite Abelian groups that a finite Abelian group whose factor algebra is Boolean must be cyclic. But for infinite Abelian groups, no such characterization of those with Boolean factor algebras is known. In

${ }^{11}$ For this result, indeed, for the more general result quoted below on lattices of subgroups, see [7], p. 267. The example of an indecomposable Abelian group which is not a generalized cyclic group, also given below, is closely related to the counterexamples provided by B. Jónsson, On Unique Factorization Problem for Torsionfree Abelian Groups, Abstract, Bull. Amer. Math. Soc. 51 (1945), 364. 
fact, there are Abelian groups which are not generalized cyclic groups and which are indecomposable (their factor algebras being thus trivially Boolean). Such, for example, is the additive group of all number pairs of the form $\left\langle 3^{n} i+m / 2\right.$, $\left.5^{n} j+m / 2\right\rangle$, where $i, j, m$ and $n$ are integers.

THEOREM 6. If, in an algebra $\mathfrak{A}=\langle A,+\rangle$, the only $\left(A, A^{c}\right)$ homomorphism (with domain restricted to $A$ ) is the constant function $f$ with $f(a)=0$ for all $a$ in $A$, then the factor algebra of 2t is Boolean. ${ }^{12}$

Proof. Let $A=B \times C$, and let $k$ be a $\left(C, B^{c}\right)$-homomorphism. As we know, there is an $(A, B)$-homomorphism $g$ and an $(A, C)$-homomorphism $h$ such that $a=g(a)+h(a)$ for all $a$ in $A$. Then $k h$ is an $\left(A, B^{c}\right)$ homomorphism. Since $B^{c}$ is known to be included ${ }^{13}$ in $A^{c}$, $k h$ is also an $\left(A, A^{c}\right)$ homomorphism, and therefore, by hypothesis, $k h(a)=0$ for all $a$ in $A$. If in particular $a \in C$, we see that $a=h(a)$, and hence $k(a)=0$. Now by applying Corollary 4 we obtain the conclusion.

Using the notion of a centerless algebra, as defined in the Introduction, we obtain as immediate consequences of Theorem 6:

COROLLARY 7. The factor algebra of a centerless algebra is Boolean.

COROLLARY 8. If $\mathfrak{A}=\langle A,+\rangle$ is either (i) a centerless group, or (ii) an algebra in which, for any $a, b$ in $A, a+b=0$ implies $a=b=0$ (in particular, if 2 is a lattice ${ }^{14}$ with zero), then the factor algebra of $\mathscr{A}$ is Boolean.

Besides centerless algebras, there is another rather comprehensive class of algebraic systems which satisfy the hypothesis of Theorem 6, which will be referred to as zero-equivalent algebras. We arrive at these algebras in the following way: ${ }^{15}$

Given an algebra $20=\langle A,+\rangle$, we define recursively the equivalence in order $n$ between two elements $a, b$ in $A$, this relationship being expressed symbolically by $a \overline{\overline{\bar{n}}} b$ :

${ }^{12}$ The results contained in Theorem 6 and its various applications mentioned below (to centerless algebras, lattices with a zero element, centerless groups, and groups which coincide with their commutator subgroups) are stated in [4], pp. 53-55; references to earlier related results (for example, of G. Birkhoff and A. Speiser) can also be found there.

${ }^{13}$ See [4], p. 26, Theorem 2.11.

${ }^{14} \mathrm{~A}$ lattice is here considered to be a system with one operation + (join operation); see [8], p. $200 \mathrm{ff}$.

${ }^{15}$ For a related construction see [0], $\$ 2$. 
(i) $a \equiv b$ if either $a=c+d$ and $b=d+c$ for some $c$ and $d$ in $A$, or else $a=c+(d+e)$ and $b=(c+d)+e$ for some $c, d$, and $e$ in $A$;

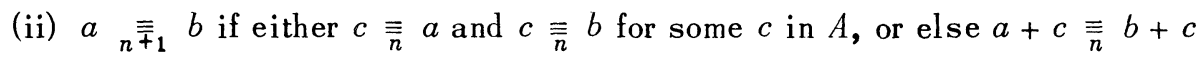
for some $c$ in $A$, or finally $a=c+e$ and $b=d+e$ and $c$ 兖 $d$ for some $c, d$, and $e$ in $A$.

The elements $a$ and $b$ are called equivalent-in symbols, $a \equiv b$-if they are equivalent in some order $n$. Remembering now the definition of a commutative semigroup as an algebra in which the commutative law, the associative law, and the cancellation law hold, we obtain the following theorem:

THEOREM 9. The relation $\equiv$ is a congruence relation over 2 ; and the coset algebra $\mathscr{N} / \equiv$ is a commutative semigroup. In fact, $\equiv$ is the smallest congruence relation $R$ for which $\mathfrak{Q} / R$ is a commutative semigroup.

Proof. It is easily shown that the relation $\equiv$ is reflexive, symmetric, and transitive; and that, if any two of the three formulae $a \equiv b, c \equiv d, a+c \equiv b+d$ hold, then the third also holds. In particular, the relation $\equiv$ is a congruence relation over $\mathfrak{A}$, and we can construct the coset algebra $\mathfrak{U} / \equiv$. From the definition of $\overline{\overline{0}}$ and $\equiv$ it follows that $a+b \equiv b+a$ and $a+(b+c) \equiv(a+b)+c$ for all $a, b$ and $c$ in $A$. This establishes the commutative and associative laws for $2 / \equiv$. Further, from $a+b \equiv a+c$ it follows by the first sentence in this proof that $b \equiv c$; this proves the cancellation law. $\mathscr{U} / \equiv$ is therefore a commutative semigroup.

To prove the last statement of the theorem, it is sufficient to show that if $R$ is a congruence relation over $\mathscr{A}$ for which $\mathscr{R} / R$ is a commutative semigroup, and $a$ and $b$ are members of $A$ for which $a \equiv b$, then we have $a R b$ (that is, $\langle a, b\rangle \in R)$. But from $a \equiv b$ follows $a \equiv \overline{\bar{n}} b$ for some nonnegative integer $n$. If $n=0$, the conclusion $a R b$ follows immediately from the definition of $\overline{\overline{0}}$ and the properties of $2 / R$. The passage from $n$ to $n+1$ is carried out in a similar manner.

The algebra 2 is called zero-equivalent if $a \equiv b$ for all $a$ and $b$ in $A$, or what amounts to the same, if $a \equiv 0$ for all $a$ in $A$. The following necessary and sufficient condition for zero-equivalence is an easy consequence of Theorem 9.

COROLLARY 10. The algebra $\mathfrak{A}$ is zero-equivalent if and only if it cannot be mapped homomorphically onto any commutative semigroup with more than one element.

We can point out many different examples of zero-equivalent algebras. For instance, as is easily seen, an algebra $\langle A,+\rangle$ is zero-equivalent if to every element $a$ in $A$ there is an element $b$ such that $a+b=b$ (or $b+a=b$ ). This 
condition will be satisfied, in particular, if in addition to the zero element 0 the algebra has an infinity element $\infty$ such that $\propto+a=\propto$ (or $a+\infty=\infty$ ) for all $a$ in $A$. The description of the latter class of algebras assumes a more familiar form if one uses the multiplicative notation; the algebras to which we refer are then systems $\mathscr{U}=\langle A, \cdot\rangle$ which have a unit element 1 with $a \cdot 1=$ $1 \cdot a=a$, and a zero element 0 with $a \cdot 0=0$ (or $0 \cdot a=0$ ) for every $a$ in $A$. Again, an algebra $\langle A,+\rangle$ satisfies the above condition for zero-equivalence if every element is idempotent, that is, $a+a=a$ for all $a$ in $A$. Another interesting example of a class of zero-equivalent algebras is provided by the groups which coincide with their commutator groups; in fact, as is seen from Corollary 10, a group is zero-equivalent if and only if it coincides with its commutator group.

THEOREM 11. The factor algebra of a zero-equivalent algebra is Boolean.

Proof. Suppose the algebra $\mathscr{U}=\langle A,+\rangle$ is zero-equivalent. Let $f$ be an $\left(A, A^{c}\right)$-homomorphism. It was remarked in the Introduction that $\left\langle A^{c},+\right\rangle$ is an Abelian group; consequently any subalgebra of $A^{c}$, in particular the subalgebra onto which $f$ maps $A$, is a commutative semigroup. Hence, by Corollary 10, $f(a)=0$ for all $a$ in $A$. The conclusion now follows by application of Theorem 6 .

As an immediate consequence of this theorem and the remarks which precede it, we obtain:

COROLLARY 12. If $\mathscr{U}=\langle A,+\rangle$ is either (i) an algebra with infinity element $\infty$ such that $\infty+a=\infty$ (or $a+\infty=\infty)$ for any a in $A$, or (ii) an algebra in which $a+a=a$ for all $a$ in $A$, or (iii) a group identical with its commutator group, then the factor algebra of $2 \mathrm{C}$ is Boolean.

Note that Corollary 12 (ii) includes, as does Corollary 8 (ii), the case of lattices with zero element.

To conclude this section, we should like to point out a rather interesting application of Theorem 1, and specifically of Corollary 2. So far we have been concerned with algebras $\mathfrak{U}=\langle A,+\rangle$ having the unique complement property, in the sense that every factor $B$ of $\mathscr{Q}$ has exactly one complementary factor. We have seen in Theorem 3 that these algebras coincide with those whose factor algebras are Boolean. We now turn to algebras which have the unique complement property in a different sense; in fact, to those algebras $\mathscr{U}=\langle A,+\rangle$ for which the set $A$ has only one complementary factor with respect to every algebra $\mathfrak{B}$ in which $\mathscr{U}$ is embedded as a subalgebra and of which $A$ is a factor. By saying that the algebra $\mathfrak{U}=\langle A,+\rangle$ is embedded as a subalgebra in the algebra $\mathfrak{B}=$ $\left\langle B,+^{\prime}\right\rangle$, we mean that $A$ is a subalgebra of $B$ in the sense of the Introduction, 
and that the operations + and $+^{\prime}$ coincide when applied to couples of elements in $A$; we assume here that the operation + is not defined for any other couples.

It has been stated that for an algebra 20 to have the unique complement property in the new sense, it is necessary and sufficient that $\mathscr{O}$ be centerless. ${ }^{16}$ The proof of this statement can easily be carried through by means of Theorem 1 and Corollary 2. In fact, the sufficiency of the condition follows almost directly from Corollary 2. To establish its necessity, we consider an arbitrary algebra $\mathscr{U}=\langle A,+\rangle$. We can easily embed $\mathfrak{A}$ as a subalgebra in a new algebra $B=$ $\left\langle B,+^{\prime}\right\rangle$ in which $B=A \times C, C$ being a subalgebra of $B$ isomorphic to $A^{c}$. Let $f$ be a function which maps $C$ isomorphically-and hence also homomorphicallyonto $A^{c}$. Then $D$, defined as the set of all $c+f(c)$, where $c \in C$, is clearly a subalgebra of $\mathscr{B}$; and we have by Theorem 1 that $B=A \times D$. Now if $\mathscr{N}$ is not centerless, $A^{c}$ contains a nonzero element; therefore $f(c) \neq 0$ for some $c$ in $C$. Hence $C \neq D$, and $A$ has two distinct complements in $\mathscr{B}$. This completes the proof. In consequence, the class of algebras having the unique complement property in the new sense is included in, but by no means identical with, the class of those algebras whose factor algebras are Boolean.

4. Algebras with many operations. ${ }^{17}$ Up to now we have considered only algebras with one binary operation. In this final section we shall state the corresponding results for a large class of algebraic systems with many operations.

A system $\mathfrak{A}=\left\langle A,+, O_{0}, O_{1}, \ldots, O_{\xi}, \ldots\right\rangle$ will be referred to as a manyoperational algebra if it is constituted by a set $A$, a binary operation + , and a finite or transfinite sequence of operations $O_{0}, O_{1}, \ldots, O_{\xi}, \ldots$. The set $A$ is assumed to be closed under all these operations, and to contain an element 0 which is the zero element for + and is idempotent for each of the remaining operations $O_{\xi}$; that is, $O_{\xi}(0,0, \ldots, 0)=0$. We restrict ourselves to the case where all the operations $O_{\xi}$ are finitary, ${ }^{\mathbf{1 8}}$ that is, each of them is defined exclusively for finite sequences of definite length $n_{\xi}$ (the number $n_{\xi}$ is called the rank of $O_{\xi}$, and varies with $\xi$ ).

By a subalgebra of 20 we understand a subset $B$ of $A$ which contains 0 and is closed under all the operations $+, O_{0}, O_{1}, \ldots, O_{\xi}, \ldots$. If $B$ and $C$ are subalgebras of $\mathscr{A}$, the definition of a $(B, C)$-homomorphism or $(B, C)$-isomorphism is an obvious extension of the earlier case. The definitions of a congruence relation $R$ over $\mathfrak{U}$ and of the coset algebra $\mathfrak{U} / R$ are also easily generalized from the earlier case.

${ }^{16}$ See [4], p. 55. The proof of this statement has not previously been published.

${ }^{17}$ See [4], $\$ \S 1$ and 2 , for most of the definitions given in this section.

${ }^{18}$ With small changes, the theory also applies to algebras with infinitary operations. 
The definition of center for many-operational algebras is somewhat involved. Given an algebra $\mathscr{A}=\left\langle A,+, O_{0}, O_{1}, \cdots, O_{\xi}, \cdots\right\rangle$, and a subalgebra $B$ of $\mathscr{N}$, by the center $B^{c}$ of $B$ we understand the union of all subalgebras $C$ of $\mathscr{A}$ which are included in $B$ and satisfy the following conditions: (i) to every element $c$ in $C$ there is an element $d$ in $C$ with $c+d=0$; (ii) the commutative-associative formula

$$
\left(b_{1}+b_{2}\right)+c=b_{1}+\left(b_{2}+c\right)=\left(b_{1}+c\right)+b_{2}
$$

holds for any $b_{1}, b_{2}$ in $B$ and any $c$ in $C$; (iii) the formula

$$
\begin{aligned}
& O_{\xi}\left(b_{0}, b_{1}, \ldots, b_{q-1}, b_{q}+c, b_{q+1}, \ldots, b_{p-1}\right) \\
= & O_{\xi}\left(b_{0}, b_{1}, \ldots, b_{p-1}\right)+O_{\xi}(0,0, \ldots, 0, c, 0, \ldots, 0)
\end{aligned}
$$

holds whenever $O_{\xi}$ is an operation of $2 \mathbb{C}$ with $p=n_{\xi}, c$ is in $C, b_{0}, b_{1}, \ldots, b_{p-1}$ are elements of $B$, and $q$ is a nonnegative integer less than $p .{ }^{19}$ It follows from this definition that $B^{c}$ is itself a subalgebra of 2 . As before, a subalgebra $B$ of 2 , or the algebra $\mathscr{Q}$ itself, is called centerless if $B^{c}=\{0\}$, or $A^{c}=\{0\}$, respectively.

If $\mathscr{U}$ is an algebra in the new sense, the notion of the direct product $D$ of two subalgebras $B$ and $C$ of $\mathscr{P}$ is defined just as it was in the Introduction for algebras with one operation, except that to conditions (i), (ii), (iii) is added a fourth condition, namely: (iv) the formula

$$
\begin{aligned}
& O_{\xi}\left(b_{0}+c_{0}, b_{1}+c_{1}, \ldots, b_{p-1}+c_{p-1}\right) \\
= & O_{\xi}\left(b_{0}, b_{1}, \ldots, b_{p-1}\right)+O_{\xi}\left(c_{0}, c_{1}, \ldots, c_{p-1}\right)
\end{aligned}
$$

holds whenever $O_{\xi}$ is an operation of $2 \mathbb{U}$ with $p=n_{\xi}, b_{0}, b_{1}, \ldots, b_{p-1}$ are elements in $B$, and $c_{0}, c_{1}, \cdots, c_{p-1}$ are elements in $C$. The concepts of a factor of $\mathfrak{X}$, of complementary factors, and of the factor algebra of $\mathfrak{A}$ are defined in terms of the direct product precisely as they were in the one-operation case.

The concepts of equivalence and zero-equivalence also have a natural extension to the case of algebras with many operations. We obtain it by modifying the definition of equivalence of order $n$ (as given in $\$ 3$ after Corollary 8 ) as follows:

(i) $a \equiv \overline{\overline{0}} b$ if either

${ }^{19}$ The definition of center just given is equivalent to Definition 2.10 of [4], p. 24, if the latter is applied to al gebras without infinitary operations. 
1) one of the two alternatives in the definition of $\overline{\overline{0}}$ in $\$ 3$ holds,

or

2) $a=O_{\xi}\left(c_{0}, c_{1}, \cdots, c_{p-1}\right)+O_{\xi}\left(d_{0}, d_{1}, \cdots, d_{p-1}\right)$ and

$$
b=O_{\xi}\left(c_{0}+d_{0}, c_{1}+d_{1}, \ldots, c_{p-1}+d_{p-1}\right)
$$

for some operation $O_{\xi}$ and some elements $c_{0}, \ldots, c_{p-1}, d_{0}, \cdots, d_{p-1}$ in $A$ (where $\left.p=n_{\xi}\right)$;

(ii) $a_{n} \overline{\overline{\bar{T}}}_{1} b$ if either

1) any one of the three alternatives in the definition of ${ }_{n} \overline{\overline{\overline{+}}}$ in $\$ 3$ holds, or

2) $a=O_{\xi}\left(c_{0}, c_{1}, \cdots, c_{p-1}\right), b=O_{\xi}\left(d_{0}, d_{1}, \cdots, d_{p-1}\right)$, and

$$
c_{i} \equiv d_{i} \quad(i=0,1, \cdots, p-1)
$$

for some $O_{\xi}$ and some elements $c_{0}, \cdots, c_{p-1}, d_{0}, \cdots, d_{p-1}$ in $A$ (where $\left.p=n_{\xi}\right)$.

As before, $a$ and $b$ are called equivalent-in symbols, $a \equiv b$ - if they are equivalent of some order $n$. The algebra $\mathscr{A}$ is said, as before, to be zero-equivalent if $a \equiv 0$ for every $a$ in $A$.

Corresponding to the notion of a commutative semigroup, which appeared in the treatment of equivalence for one-operation algebras, here we need the notion of a commutative semigroup with operators, defined as follows: $\mathscr{A}=\langle A,+$, $\left.O_{0}, O_{1}, \cdots, O_{\xi}, \cdots\right\rangle$ is a commutative semigroup with operators if $(\mathrm{i})\langle A,+\rangle$ is a commutative semigroup, and (ii) the relation

$$
\begin{aligned}
& O_{\xi}\left(a_{0}, a_{1}, \ldots, a_{p-1}\right)+O_{\xi}\left(b_{0}, b_{1}, \ldots, b_{p-1}\right) \\
= & O_{\xi}\left(a_{0}+b_{0}, a_{1}+b_{1}, \ldots, a_{p-1}+b_{p-1}\right)
\end{aligned}
$$

holds for all $O_{\xi}$ and all elements $a_{0}, \cdots, a_{p-1}, b_{0}, \cdots, b_{p-1}$ of $A$ (where $\left.p=n_{\xi}\right)$.

Having extended the basic notions, one can carry through for many-operational algebras a development similar to that given in $\$ 2$ and $\$ 3$ for algebras with one operation. One then obtains:

THEOREM 13. Theorems and Corollaries $1,2,3,4,6,7$ and 11 remain valid for many-operational algebras. Theorem 9 and Corollary 10 hold for many-oper- 
ational algebras if in them we replace 'commutative semigroup' by 'commutative semigroup with operators.'

Observe that in the definition of many-operational algebras the first operation + plays a special role. On the other hand, it may happen that an algebra $\mathscr{U}=$ $\left\langle A,+, O_{0}, O_{1}, \cdots, O_{\xi}, \cdots\right\rangle$ contains a binary operation $O_{\xi}$ which has a zero element that is idempotent with respect to all remaining operations (+ included). By permuting, then, the operations $+, O_{0}, O_{1}, \cdots, O_{\xi}, \cdots$ so that $O_{\xi}$ replaces + , we obtain a new algebra $\mathfrak{U}^{\prime}$ which is formally different from $\mathscr{U}$. It turns out, however, that if the factor algebra of $\mathscr{C}$ is Boolean, the same holds for $\mathfrak{U}^{\prime}$. Also it turns out that if the factor algebra of $\mathscr{U}$ is Boolean, the same applies to every algebra $2{ }^{\prime}$ 'obtained from \&f by adding new operations. These two facts are combined in the following theorem:

THEOREM 14. Suppose that \& $=\left\langle A,+, O_{0}, O_{1}, \cdots, O_{\xi}, \cdots\right\rangle$ and $\mathscr{U}^{\prime}=$ $\left\langle A,+^{\prime}, O_{0}^{\prime}, O_{1}^{\prime}, \cdots, O_{\eta}^{\prime}, \cdots\right\rangle$ are two many-operational algebras (having the same set $A)$, and that the family $\left\{+^{\prime}, O_{0}^{\prime}, O_{1}^{\prime}, \cdots, O_{\eta}^{\prime}, \cdots\right\}$ of operations of $\mathfrak{A}^{\prime}$ contains the family $\left\{+, O_{0}, O_{1}, \cdots, O_{\xi}, \ldots\right\}$ of operations of $\mathcal{X}$. If the factor algebra of $\mathfrak{Q}$ is Boolean, then that of $\mathfrak{U}^{\prime}$ is also Boolean. 20

Proof. The symbols $x$ and $x^{\prime}$ will refer to the operation of direct multiplication in the algebras $\mathscr{U}$ and $\mathfrak{U}^{\prime}$, respectively. Let 0 and $0^{\prime}$ be the zero elements of + and $t^{\prime}$, respectively.

We define an operation * on the factors of $\mathscr{C}^{\prime}$ as follows: Let $B$ be any factor of 'श', and let $C$ be some complementary factor of $B$ (in $\mathscr{Y}^{\prime}$ ). Let $0_{B}$ and $0_{C}$ be such elements of $B$ and $C$ respectively that $0=0_{B}+{ }^{\prime} 0_{C}$. Then $B^{*}$ is defined to be the set of all elements of the form $b+{ }^{\prime} 0_{C}$ with $b$ in $B$.

Notice that, in case there are several complementary factors of $B$ in $\mathscr{U}^{\prime}$, the definition of $B^{*}$ is independent of which one is chosen as $C$.

We now fix two complementary factors $B$ and $C$ in $\mathfrak{Y}^{\prime}$. We shall show that $B^{*}$ and $C^{*}$ are complementary factors in $\mathscr{U}$. As before, let $0_{B}$ and $0_{C}$ be such elements of $B$ and $C$ respectively that

$$
0=0_{B}+{ }^{\prime} 0_{C}
$$

First observe that

$$
0 \in B^{*} \cap C^{*}
$$

${ }^{20}$ This theorem is a simple corollary of some more general results obtained by $B$. Jónsson and A. Tarski but not yet published, concerning algebraic systems which do not necessarily have a zero element. 
Let $P$ be any one of the operations in $\mathfrak{A}$, of rank $p$; by the hypotheses of the theorem, $P$ is also an operation in $2^{\prime}$. From the idempotency of 0 with respect to $P$ and the well-known properties of the direct product (which in future we use without mention), we deduce that

$$
P\left(0_{B}, 0_{B}, \cdots, 0_{B}\right)=0_{B}
$$

and

$$
P\left(0_{C}, 0_{C}, \cdots, 0_{C}\right)=0_{C} \text {. }
$$

Furthermore, if $b_{0}, \cdots, b_{p-1} \in B$, we obtain from (3) the result that

$$
P\left(b_{0}+{ }^{\prime} 0_{C}, \cdots, b_{p-1}+{ }^{\prime} 0_{C}\right)=P\left(b_{0}, \cdots, b_{p-1}\right)+{ }^{\prime} 0_{C} ;
$$

similarly, if $c_{0}, \cdots, c_{p-1} \in C$, we have

$$
P\left(c_{0}+0_{B}, \cdots, c_{p-1}+0_{B}\right)=P\left(c_{0}, \cdots, c_{p-1}\right)+{ }^{\prime} 0_{B} \cdot
$$

Now (1), (4), and (5) give that

$$
B^{*} \text { and } C^{*} \text { are subalgebras of } \mathscr{2} \text {. }
$$

From the fact that 0 is the zero element of + in $A$, it is not hard to show that $0_{B}$ and $0_{C}$ are the zero elements of + in $B$ and $C$ respectively; that is,

$$
b+0_{B}=0_{B}+b=b \text { for all } b \text { in } B
$$

and

$$
c+0_{C}=0_{C}+c=c \text { for all } c \text { in } C .
$$

Using (7) and (8) we find that

$$
\left(b+{ }^{\prime} 0_{C}\right)+\left(c+{ }^{\prime} 0_{B}\right)=b++^{\prime} c \text { whenever } b \in B, c \in C,
$$

from which it follows that

(10) every element of $A$ can be written uniquely in the form $b^{*}+c^{*}$, with $b^{*}$ in $B^{*}$ and $c^{*}$ in $C^{*}$.

Further, if $P$ is any operation of 2, , of rank $p$, and if

$$
b_{0}, \ldots, b_{p-1} \in B ; c_{0}, \ldots, c_{p-1} \in C,
$$

then by repeated application of (9), (4), and (5), we get

$$
\begin{aligned}
& P\left\{\left[\left(b_{0}+0_{C}\right)+\left(c_{0}+^{\prime} 0_{B}\right)\right], \cdots,\left[\left(b_{p-1}+{ }^{\prime} 0_{C}\right)+\left(c_{p-1}+{ }^{\prime} 0_{B}\right)\right]\right\} \\
& =P\left[b_{0}+0_{C}, \cdots, b_{p-1}+' 0_{C}\right]+P\left[c_{0}+{ }^{\prime} 0_{B}, \cdots, c_{p-1}+' 0_{B}\right] .
\end{aligned}
$$


Now (6), (10), and (11) give

$$
B^{*} \times C^{*}=A \text {. }
$$

Thus we have shown that $B^{*}$ and $C^{*}$ are complementary factors in $2 \mathbb{C}$ whenever $B$ and $C$ are complementary factors in $2{ }^{\prime}$.

Now let $B, C_{1}$, and $C_{2}$ be such subalgebras of $\mathscr{Q}^{\prime}$ that

$$
B \times{ }^{\prime} C_{1}=B \times{ }^{\prime} C_{2}=A \text {. }
$$

By our last result, we have

$$
B^{*} \times C_{1}^{*}=B^{*} \times C_{2}^{*}=A .
$$

But, since the factor algebra of 21 is by hypothesis Boolean, (13) combined with Theorem 3 (see Theorem 13) gives

$$
C_{1}^{*}=C_{2}^{*} .
$$

Now using Theorem 1 (see Theorem 13), let $g$ be the $\left(C_{1}, B^{c}\right)$-homomorphism with respect to $\mathscr{P}^{\prime}\left(B^{c}\right.$ being the center of $B$ in $\mathscr{Q}^{\prime}$ ) such that $C_{2}$ is the set of elements of the form $c_{1}+^{\prime} g\left(c_{1}\right)$ with $c_{1}$ in $C_{1}$. Let $0_{B}$ and $0_{B}^{+}$be those elements of $B$ for which, for some $0_{C}$ in $C_{1}$ and $0_{C}^{+}$in $C_{2}$,

$$
0=0_{B}+0_{C}=0_{B}^{+}+0_{C}^{+} \text {. }
$$

Then (14) together with (12) assures us that

$$
c_{1}+{ }^{\prime} 0_{B}=c_{1}+{ }^{\prime}\left[g\left(c_{1}\right)+{ }^{\prime} 0_{B}^{+}\right] \text {for all } c_{1} \text { in } C_{1} \text {. }
$$

In particular, setting $c_{1}=0^{\prime}$ in (15), we find

$$
0_{B}=0_{B}^{+} ;
$$

hence (15) assumes the form

$$
0_{B}=g\left(c_{1}\right)+{ }^{\prime} 0_{B} \text { for all } c_{1} \text { in } C_{1} \text {. }
$$

But by the definition of $g$, we have $g\left(c_{1}\right) \in B^{c}$ for each $c_{1}$ in $C_{1}$; consequently

$$
\begin{aligned}
& b_{1}+\left[b_{2}++^{\prime} g\left(c_{1}\right)\right]=\left(b_{1}+b_{2}\right)+{ }^{\prime}\left[0^{\prime}+g\left(c_{1}\right)\right] \\
& \text { whenever } b_{1}, b_{2} \in B \text { and } c_{1} \in C_{1} .
\end{aligned}
$$

Setting $b_{2}=0_{B}$ and $b_{1}=0^{\prime}$ in (17) we conclude with the aid of (16) and (7) that

$$
0^{\prime}+g\left(c_{1}\right)=0^{\prime} \text { for each } c_{1} \text { in } C_{1} \text {. }
$$


On the other hand, setting $b_{1}=0_{B}$ and $b_{2}=0^{\prime}$ in (17), we get with the aid of (18) and (7) the result that

$$
g\left(c_{1}\right)=0^{\prime} \text { for all } c_{1} \text { in } C_{1} \text {. }
$$

Consequently, by the definition of $g$, we have

$$
C_{1}=C_{2} \text {. }
$$

We have now shown that if $B, C_{1}$, and $C_{2}$ are subalgebras of $x^{\prime}$ for which (12) holds, then $C_{1}=C_{2}$. But, by Theorem 3 , this implies that the factor algebra of $2^{\prime}$ is Boolean.

We shall end this section with an application of the general theory to rings.

THEOREM 15. The factor algebra of a ring with unit element is Boolean. ${ }^{21}$

We can prove this theorem by three different methods, applying either Corollary 7, Theorem 11, or Theorem 14 .

First proof. Let $\mathscr{Y}^{\prime}=\langle A,+, \cdot\rangle$ be a ring with the unit element 1 (and of course with the zero element 0). Let $c \in A^{c}$. By the definition of center, we must have

$$
(a+c) \cdot b=a \cdot b \text { for all } a, b \text { in } A .
$$

Setting $a=0, b=1$, we obtain $c=0$. Thus $\mathfrak{I}^{\prime}$ is centerless, and by applying Corollary 7 (see Theorem 13) we obtain the desired result.

Second proof. Let $\mathfrak{x}^{\bullet}=\langle A,+, \cdot\rangle$ be a ring with unit element 1 . By the definition of equivalence of order 0 , we have

$$
(a+c) \cdot(b+d) \equiv a \cdot b+c \cdot d \text { for all } a, b, c, d \text { in } A .
$$

Setting $b=c=0$ and $d=1$, we obtain $a \equiv 0$ for all $a$ in $A$. $\mathbb{X}^{\prime}$ is therefore zeroequivalent, and the desired result follows from Theorem 11 (see Theorem 13).

Third proof. Again, let $\mathscr{A}^{\prime}=\langle A,+, \cdot\rangle$ be a ring with unit. Let $\mathfrak{A}=\langle A, \cdot\rangle$. By Corollary 12 (and a remark following Corollary 10) the factor algebra of $\mathscr{A}$ is Boolean. Hence, by Theorem 14, the factor algebra of $\mathfrak{Y}^{\prime}$ is Boolean.

It may be noticed that, by applying the first two methods of proof, we can extend Theorem 15 to wider classes of rings. In fact, the first method of proof

${ }^{21}$ This result is stated in a different form in [4], p. 55, where a reference to an earlier result of Jacobson can be found. The extension of this result to centerless rings, which will be discussed below, is to be found in [6]; it also follows immediately from remarks in [4], p. 25 (Example III) and p. 54. 
permits us to extend it to all centerless rings, ${ }^{22}$ while by the second method of proof it applies to all zero-equivalent rings. It is easily seen that a ring is centerless if and only if it has no annihilator different from 0 , that is, no element $a \neq 0$ such that $a \cdot b=b \cdot a=0$ for all elements $b$ of the ring. On the other hand a ring proves to be zero-equivalent if and only if every element $a$ of the ring is of the form

$$
a=b_{0} \cdot c_{0}+b_{1} \cdot c_{1}+\cdots+b_{n-1} \cdot c_{n-1},
$$

where $n$ is a positive integer and $b_{0}, c_{0}, b_{1}, c_{1}, \cdots, b_{n-1}, c_{n-1}$ are elements of the ring. Either of these classes includes all rings with unit and also many other rings.

It may be noticed that neither of these two classes of rings includes the other. Indeed, the ring of even integers is centerless but not zero-equivalent. To obtain a ring which is zero-equivalent but not centerless, consider the family $R$ of all finite sets of real numbers $x$ for which $0<x \leq 1$. If $A, B \in R$, we denote by $A+B$ the symmetric difference of $A$ and $B$, that is, the set of numbers belonging either to $A$ or to $B$ but not to both; and we denote by $A \cdot B$ the set of all numbers $x$ for which (i) $0<x \leq 1$ and (ii) the number of elements $y$ of $A$ such that $x-y \in B$ is odd. The system $\Re=\langle R,+, \cdot\rangle$ can be shown to be a ring which is zero-equivalent but not centerless. Indeed, $\Re$ has the further properties that it is commutative and every element in it is of order 2.

Examples of rings whose factor algebras are not Boolean are easy to obtain. Let $\mathscr{U}=\langle A,+\rangle$ be an Abelian group with zero element 0 , and set $a \cdot b=0$ for all $a, b$ in $A$. Then $\mathscr{X}^{\circ}=\langle A,+, \cdot\rangle$ is a ring whose factors are identical with those of $\mathscr{X}$. Thus, if the factor algebra of $\mathscr{U}$ is not Boolean (for example, if $\mathscr{U}$ is the four group, that is, the direct product of two groups of order 2), then the factor algebra of $\mathfrak{2}^{\prime}$ also is not Boolean.

\section{REFERENCES}

1. R. Baer, Direct decompositions, Trans. Amer. Math. Soc. 62 (1947), 62-98.

2. G. Bergman, Zur Axiomatik der Geometrie, Monatsh. Math. Phys. 36 (1929), 269 284.

3. G. Birkhoff and S. MacLane, A survey of modern algebra, Macmillan, 1941.

${ }^{22}$ It should be noticed that our definition of the center of a ring, obtained by specializing to rings the general definition of the center of an algebra with many operations, differs from the usual meaning of that term. 
4. B. Jónsson and A. Tarski, Direct decompositions of finite algebraic systems, Notre Dame Mathematical Lectures, No. 5, 1947.

5. - On direct products of algebras, Abstract 149, Bull. Amer. Math. Soc. $51(1945), 656$.

6. A. Kurosh, Izomorfizmy pryamyh razlozenii (Isomorphisms of direct decompositions), (Russian; English Summary), Izvestiya Akad. Nauk S.S.S.R. Ser. Mat. 7 (1943), $185-202$.

7. O. Ore, Structures and group theory, II, Duke Math. J., 4 (1938), 247-269.

8. A. Tarski, Cardinal algebras, with an appendix by B. Jónsson and A. Tarski, Cardinal products of isomorphism types, New York, 1949.

9. A. Tarski, Algebraische Fassung des Massproblems, Fund. Math., 31 (1938), 47-66.

University of California, Ber Keley 


\title{
PACIFIC JOURNAL OF MATHEMATICS
}

\section{EDITORS}

\section{R. M. RobINSON \\ University of California \\ Berkeley 4, California}

\author{
*R. P. Dilwor TH \\ California Institute of Technology \\ Pasadena 4, California
}

\author{
E. F. BECKENBACH, Managing Editor \\ University of California \\ Los Angeles 24, California
}

${ }^{*}$ During the absence of Herbert Busemann in 1952.

\section{ASSOCIATE EDITORS}

$\begin{array}{llll}\text { R. P. DILWORTH } & \text { P. R. HALMOS } & \text { B } \emptyset \text { RGE JESSEN } & \text { J. J. STOKER } \\ \text { HERBERT FEDERER } & \text { HEINZ HOPF } & \text { PAUL LÉVY } & \text { E. G. STRAUS } \\ \text { MARSHALL HALL } & \text { R. D. JAMES } & \text { GEORGE PÓLYA } & \text { KÖSAKU YOSIDA }\end{array}$

\section{SPONSORS}

UNIVERSITY OF BRITISH COLUMBIA

CALIFORNIA INSTITUTE OF TECHNOLOGY

U NIVERSITY OF CALIFORNIA, BERKELEY

UNIVERSITY OF CALIFORNIA, DAVIS

UNIVERSITY OF CALIFORNIA, LOS ANGELES

UNIVERSITY OF CALIFORNIA, SANTA BARBARA

OREGON STATE COLLEGE

UNIVERSITY OF OREGON

\author{
UNIVERSITY OF SOUTHERN CALIFORNIA \\ STANFORD UNIVERSITY \\ WASHINGTON STATE COLLEGE \\ UNIVERSITY OF WASHINGTON \\ AMERICAN MATHEMATICAL SOCIETY \\ NATIONAL BUREAU OF STANDARDS, \\ INSTITUTE FOR NUMERICAL ANALYSIS
}

Mathematical papers intended for publication in the Pacific Journal of Miathematics should be typewritten (double spaced), and the author should keep a complete copy. Manuscripts may be sent to any of the editors. All other communications to the editors should be addressed to the managing editor, E. F. Beckenbach, at the address given above.

Authors are entitled to receive 100 free reprints of their published papers and may obtain additional copies at cost.

The Pacific Journal of Mathematics is published quarterly, in March, June, September, and December, by the University of California, Berkeley 4, California. The price per volume (4 numbers) is $\$ 8.00$; single issues, $\$ 2.50$. Special price to individual faculty members of supporting institutions and to individual members of the American Mathematical Society: $\$ 4.00$ per volume; single issues, $\$ 1.25$.

Subscriptions, orders for back numbers, and changes of address should be sent to the publishers, University of California Press, Berkeley 4, California.

Printed at Ann Arbor, Michigan. Entered as second class matter at the Post Office, Berkeley, California.

\section{UNIVERSITY OF CALIFORNIA PRESS • BERKELEY AND LOS ANGELES}




\section{Pacific Journal of Mathematics}

\section{Vol. 2, No. $3 \quad$ March, 1952}

Lars V. Ahlfors, Remarks on the Neumann-Poincaré integral equation .... 271

Leonard P. Burton, Oscillation theorems for the solutions of linear, nonhomogeneous, second-order differential systems ............ 281

Paul Civin, Multiplicative closure and the Walsh functions . . . . . . . . . . . 291

James Michael Gardner Fell and Alfred Tarski, On algebras whose factor algebras are Boolean .................................. 297

Paul Joseph Kelly and Lowell J. Paige, Symmetric perpendicularity in Hilbert geometries .................................. 319

G. Kurepa, On a characteristic property of finite sets .............. 323

Joseph Lehner, A diophantine property of the Fuchsian groups ......... 327

Donald Alan Norton, Groups of orthogonal row-latin squares ........... 335

R. S. Phillips, On the generation of semigroups of linear operators ....... 343

G. Piranian, Uniformly accessible Jordan curves through large sets of relative harmonic measure zero ........................ 371

C. T. Rajagopal, Note on some Tauberian theorems of $O . S z \tilde{A} ; s z \ldots \ldots \ldots 377$

Halsey Lawrence Royden, Jr., A modification of the Neumann-Poincaré method for multiply connected regions .................... 385

George H. Seifert, A third order irregular boundary value problem and the associated series ...................................... 395

Herbert E. Vaughan, Well-ordered subsets and maximal members of ordered sets....

Hans F. Weinberger, An optimum problem in the Weinstein method for eigenvalues.

Shigeki Yano, Note on Fourier analysis. XXXI. Cesàro summability of Fourier series. 